\title{
Educação superior e crescimento pessoal: motivações sociais entre personagens nucleares do meio universitário
}

\author{
José Augusto Dela Coleta \\ Marilia Ferreira Dela Coleta
}

\begin{abstract}
Resumo
Baseando-se nos resultados de quase duas dezenas de estudos conduzidos com sujeitos brasileiros, todos personagens nucleares relacionados ao meio universitário (professores, candidatos, alunos, egressos), envolvendo variáveis contidas nos modelos de cultura maior (social) e de cultura menor (industrial), propostos por Romero-Garcia para explicar o processo motivacional de indivíduos vivendo em sociedades em vias de desenvolvimento, este trabalho evidencia a consistência teórica dos modelos propostos. A análise destes estudos aponta para a importância do nível de escolaridade e socioeconômico, do ambiente motivacional do lar e do porte da cidade onde vivem os sujeitos para a caracterização psicossocial de sua cultura.

Palavras-Chave: Cultura; Motivações sociais; Estudantes; Professores.
\end{abstract}

\section{Higher education and personal development: Social motivations among nuclear people from university}

\begin{abstract}
Based on the results of almost twenty studies developed with Brazilian subjects, all nuclear people from university (professors, applicants, students, graduated), and using the variables included in the societal culture model and industrial culture model, proposed by Romero-Garcia to explain the motivational processes of individuals living in developing societies, this work shows the theoretical consistency of the proposed models. The analysis of these studies indicate the importance of the educational and economical level, the motivational environment at home, and the size of the city where people live, to the psycho-social characterization of their culture.
\end{abstract}

Keywords: Culture; Social motivations; Students; Professors.

\section{Introdução}

Em meados dos anos 70, o Centro de Investigações Psicológicas da Universidade de Los Andes, em Mérida, na Venezuela, por meio de um pequeno e extremamente produtivo grupo de pesquisadores, liderados pelo dr. Oswaldo Romero-Garcia, iniciou o desenvolvimento de um vasto programa de pesquisas envolvendo a relação entre grande número de variáveis psicossociais e diversas conseqüências em situações de educação e trabalho (Romero-Garcia, 1986).

Sustentados principalmente pelos estudos sobre as motivações sociais e sua relação com o desenvolvimento dos povos (McClelland, 1972), o construto lócus de controle (Levenson, 1974; Rotter, 1966), o desamparo e o otimismo (Seligman, 1977, 1991), o sentimento de auto-estima (Coopersmith, 1967; Rosemberg, 1965), Romero-García e seus colaboradores desenvolveram centenas de pesquisas, no meio laboral e acadêmico.

Esses estudos culminaram com a proposta de modelos visando explicar a conduta do homem em situação escolar, no trabalho e na vida pessoal, principalmente na cultura latino-americana, subdesenvolvida, como sendo determinada por dois conjuntos de forças antagônicas: por um lado, pelas motivações presentes na chamada "cultura maior", definida como aquela da sociedade em geral, plena de crenças e condutas de adaptação ao subdesenvolvimento e, por outro, pelas variáveis motivacionais presentes na "cultura menor", definida como a cultura industrial e empresarial, tida como desenvolvida e presente em algumas ilhas de excelência existentes neste meio carente de desenvolvimento (Romero-Garcia, 1991).

No modelo de cultura maior, tal como definido por Romero-Garcia (1991), existiria uma série de crenças, hábitos, regras sociais, ensinamentos, que terminariam por levar as pessoas que a ele se submetem a apresentarem uma série de características compreendendo crenças referentes a que:

a) tudo no mundo já está determinado a priori; as coisas são como são, obras do destino e do acaso, a cada um estando reservado um script, contra o qual pouco ou quase nada se pode fazer (alto lócus de controle - externalidade autêntica ou acaso);

b) o alcance de qualquer objetivo depende da ajuda de outras pessoas em posições superiores (alto lócus de controle - externalidade defensiva ou "outros poderosos");

${ }^{1}$ Endereço para correspondência:

E-mail: fapsi@ufu.br 
c) o ser humano é apenas uma vítima das circunstâncias que se lhe apresentam ao longo da vida, não lhe sendo facultado intervir sobre o meio e os eventos nos quais tem interesse (baixo lócus de controle internalidade);

d) o alcance dos resultados não depende diretamente dos esforços despendidos para consegui-los (baixa esperança ativa);

e) não se deve esperar, de forma racional, que o melhor sempre venha a ocorrer (baixo otimismo);

f) as opiniões sobre diferentes aspectos da vida em sociedade não merecem tanto empenho para que sejam preservadas, assim como não se deve levar o respeito à própria pessoa às últimas conseqüências, não defendendo firmemente suas posições, não se esforçando para fazer valer o que pensa (baixa assertividade);

g) a pessoa não se vê como um indivíduo valioso, com conceitos positivos sobre si mesmo, capaz de se orgulhar de suas realizações, de seus princípios, de suas qualidades (baixo sentimento de auto-estima);

h) o sujeito é carente de preocupações em alocar forças para enfrentar com coragem e dignidade as adversidades e dificuldades que a vida apresenta (baixa fortaleza pessoal);

i) o indivíduo demonstra insuficiente empenho em tarefas envolvendo a superação pessoal, o crescimento, a realização, pouco ou quase nunca estabelecendo metas controláveis e desafiadoras (baixa motivação de realização-metas);

j) existe pequena preocupação com o esforço, persistência, a definição de estratégias de ação mais adaptadas para o alcance das metas fixadas (baixa motivação de realização-instrumentação);

k) não se valoriza a necessidade de fazer o melhor o tempo todo, não se exige perfeição e qualidade, não se impõem níveis de excelência nos resultados e na forma de se comportar (baixa excelência);

1) não importa o tempo gasto na realização das tarefas, não se valoriza o tempo como elemento escasso e finito (baixa eficiência);

m) o conhecimento e a formação do indivíduo têm data para serem completados, que uma vez obtido um diploma, não mais se necessita buscar aprofundamento sobre um tema qualquer, que a escola, no sentido amplo, é um lugar para jovens até que se graduem ou se casem, depois disto não sendo mais necessário estudar (baixa especialização);

n) as pessoas tendem a apresentar orientações para a busca e valorização do poder, não nos aspectos altruístas, de auxílio desinteressado ao próximo (baixo poder benigno ou socializado);

o) tende-se a apresentar altos níveis de orientação para o poder, principalmente naqueles aspectos voltados para o poder manipulador, impositivo, que usa as pessoas em benefício próprio (alto poder explorador);

p) neste ambiente há um predomínio de tendências em procurar se dar bem com todos, em atender os interesses de cada um, mesmo em detrimento do alcance das metas fixadas (alta motivação de afiliação);

q) se busca transformar o ambiente de trabalho em um ambiente familiar, de compadrio, onde necessariamente todos devem ser amigos, mesmo às custas da eficiência do sistema (alta motivação de afiliação no trabalho);

r) se utilizem as amizades citadas no item anterior para se conseguir o que se deseja, para perseguir os inimigos e desafetos, pelo afeto manipulador, pela chantagem afetiva (alta motivação de afiliação exploradora).

Existe, entretanto, um outro mundo diferente, oposto a este anteriormente descrito, que se caracteriza pelo modelo de cultura menor que preconiza ser possível ao ser humano, em muitas circunstâncias, lutar pelos seus objetivos, superar suas limitações, alcançar a plena realização, o crescimento, o desenvolvimento como ser completo, a superação do subdesenvolvimento e a apresentar outro conjunto de características pessoais diferente.

Os resultados de pesquisas com milhares de sujeitos, na Venezuela e em outros países da América Latina, de ambos os sexos, de diversas faixas etárias, de variados níveis de escolaridade, trabalhadores, estudantes de diferentes níveis e séries acadêmicas, puderam demonstrar inequivocamente a relação entre a maior ou menor presença desses traços psicossociais e os resultados no trabalho e na escola. Assim, foi possível concluir que maior rendimento acadêmico e/ou desempenho superior no trabalho estão associados a melhor aderência ao perfil de cultura menor, com maior internalidade do lócus de controle, sentimento de auto-estima, otimismo, esperança ativa, assertividade, motivação de realização, busca de especialização, excelência, preocupação com a eficiência, grau de elaboração lingüística, valor incentivo dos estudos (disposição para iniciação dos estudos, persistência nos estudos, desafiliação nas atividades escolares), presença de crenças positivas sobre o trabalho, bem como a menores níveis de motivação de poder explorador, motivação de afiliação no trabalho, afiliação exploradora, crenças inibidoras da realização, crenças no acaso, azar, destino, crenças em outros poderosos.

Valendo-se de tais pressupostos, este trabalho tem o objetivo de sumariar diversos estudos realizados no meio brasileiro, envolvendo personagens nucleares relacionados ao meio universitário (candidatos, alunos, professores e egressos), com o intuito de oferecer subsídios à melhor compreensão das características motivacionais desta parcela da população, bem como dos efeitos da educação superior sobre o perfil dos indivíduos.

Psico-USF, v. 10, n. 1, p. 69-75, jan./jun. 2005 


\section{Resultados de pesquisas brasileiras com indivíduos relacionados ao meio universitário envolvendo variáveis componentes dos modelos de "cultura maior" e "cultura menor"}

\section{Estudos envolvendo estudantes}

Talvez no primeiro estudo conduzido no meio brasileiro envolvendo o construto "lócus de controle" e a escala de Rotter (1966), Dela Coleta (1979) encontrou resultados médios iguais a 10,54 , para um escore variando entre zero e 23 pontos, em um grupo de alunos do sexo masculino do curso de Engenharia, resultado semelhante àqueles obtidos com universitários norte-americanos e indicativo de internalidade do lócus de controle apenas um pouco superior à média.

Utilizando a escala multidimensional de Levenson (1974) para a medida do lócus de controle, M. F. Dela Coleta $(1987,1988)$ demonstrou que os alunos com nível médio de escolaridade apresentavam maiores escores no lócus de controle outros poderosos e acaso que os alunos universitários, sem diferença entre os grupos no que se refere à internalidade, mostrando ambos os grupos resultados mais altos que a média esperada em internalidade e mais baixos em externalidade acaso e outros poderosos.

Ao estudar uma amostra de 258 alunos de um curso particular preparatório aos exames de seleção para ingresso em uma Universidade Federal (Dela Coleta, J. A., 1988, 1989), foram encontrados resultados indicando maior tendência à auto-estima elevada, escores mais altos em lócus de controle internalidade e mais baixos em outros poderosos e acaso, mais alto valor incentivo dos estudos, tanto em iniciação como em persistência e desafiliação, escores superiores em motivação de realização e maiores probabilidades subjetivas de aprovação nos exames. Os dados mostraram correlações positivas entre internalidade, valor incentivo dos estudos, motivação de realização, probabilidade subjetiva de aprovação e resultado final no vestibular, e correlações negativas destas duas últimas variáveis com o lócus de controle externalidade outros poderosos e acaso. Mais importante ainda, foi encontrada correlação múltipla, significativa do ponto de vista estatístico, entre as variáveis psicossociais e o resultado final nos exames vestibulares.

Trabalhando com 425 estudantes de ambos os sexos, de universidades públicas e particulares e de escolas de ensino médio do interior de Minas Gerais, Dela Coleta e Paula (1998) encontraram evidências de que esses sujeitos tendem a apresentar níveis mais elevados de autoestima, internalidade do lócus de controle motivação de realização e valor incentivo dos estudos, encontrando-se, também, maior dificuldade para começar a estudar do que para manter este comportamento. Tomando-se a amostra total como um só grupo, demonstraram que o rendimento acadêmico dos alunos depende de maior valor incentivo dos estudos, principalmente em seus aspectos de iniciar e persistir nos estudos, do motivo de realização, auto-estima e de baixos níveis no lócus de controle externo. Uma análise geral desses resultados permitiu, ainda, concluir que as dificuldades relativas a iniciar a atividade de estudar, a facilidade em interromper a atividade por sono, preguiça ou cansaço e a perder a concentração no estudo por causa de pensamentos de afiliação, entre as variáveis estudadas, são os principais responsáveis pelas notas baixas dos estudantes, vindo a seguir a percepção de ser controlados por fatores externos, aleatórios e imprevisíveis.

Dela Coleta e outros (Dela Coleta, Marcondes, Moreira, Alvarenga, Amaral \& Santos, 2003) desenvolveram estudo comparando algumas características psicossociais de 52 alunos de cursos pré-vestibulares pagos e 99 de cursos gratuitos, verificando haver diferenças quanto à preferência de área. A maioria dos alunos do curso gratuito preferia as ciências humanas, apresentando média de idade mais alta, menor percepção de controle interno da própria vida, maior crença no controle por pessoas poderosas e pior estimação da probabilidade de obter uma vaga para o curso escolhido, sem diferença significativa quanto à auto-estima, motivo de realização e crença no controle pelo acaso.

Neste estudo mostrou-se ainda que a probabilidade subjetiva de ser aprovado no vestibular correlacionouse positivamente com motivação para a realização e auto-estima e negativamente com crenças em outros poderosos. Tais resultados indicam que os alunos do curso gratuito são tão motivados para alcançar suas metas de realização quanto os alunos do curso pago, mas que, além das diferenças econômicas, apresentam auto-confiança mais baixa e percebem grande poder de outras pessoas na determinação de suas vidas, podendo estas crenças virem a prejudicar o desempenho desses estudantes no vestibular.

Ao estudar as características psicossociais de 110 universitários considerados por seus professores como "exitosos" e 105 como "não exitosos", Dela Coleta, Almeida e Borges e Dela Coleta (2003) demonstraram que os alunos "exitosos" apresentam índices superiores de auto-estima, esperança ativa, otimismo, internalidade, eficiência, excelência, especialidade, fortaleza e assertividade, e menores escores relacionados à afiliação, ao poder explorador, crenças em outros poderosos, crenças no acaso e crenças inibidoras da realização. O trabalho concluiu também que os indivíduos que mais se aproximam do modelo de cultura menor apresentam maiores chances de obter êxito nas atividades acadêmicas de nível superior (ver Almeida \& Borges, 2001).

Em sua dissertação de mestrado, Souza (2002) examinou 427 alunos e professores de escolas de ensino médio e 24 alunos e professores universitários do 
interior do Pará, encontrando resultados mais próximos do modelo de cultura menor. Os sujeitos apresentaram elevados índices em motivação de realização, fortaleza, sentimento de auto-estima, internalidade, esperança ativa, e mais baixos em lócus de controle acaso e outros poderosos, poder explorador, afiliação exploradora e crenças negativas sobre o trabalho, além de uma tendência geral a melhores resultados entre o grupo de universitários quando comparados aos sujeitos de nível médio. Chama a atenção nos dados deste estudo o renitente alto nível de afiliação, tanto geral quanto no trabalho, em ambos os grupos examinados.

Ao mensurar diversas variáveis psicossociais de 404 alunos do curso de Administração, de uma IES privada do interior de Minas Gerais, Dela Coleta e Gomide (2003) obtiveram resultados indicando maior aproximação com o modelo de cultura menor. Os escores médios dos sujeitos mostraram-se mais altos em motivação para realização metas e instrumentação, poder benigno, auto-estima, internalidade, fortaleza, assertividade, esperança ativa, otimismo e crenças positivas relativas ao trabalho, e mais baixos em poder explorador, afiliação exploradora, crenças em outros poderosos, no acaso e crenças inibidoras da realização. Chamaram a atenção, nesta pesquisa, os altos escores médios tanto em afiliação geral, como em afiliação no trabalho, que parecem constituir-se em características do povo brasileiro e que podem comprometer uma maior aproximação com o modelo de cultura menor.

Em um outro estudo (Melo, Dela Coleta \& Dela Coleta, 2003) foram analisadas as mesmas características psicossociais de 574 universitários, sendo 311 de um centro desenvolvido (290 mil habitantes) e 263 da mesma universidade, de um pólo em desenvolvimento (35 mil habitantes). Os resultados obtidos mostram que os alunos do centro desenvolvido possuem maior esperança ativa, poder explorador e poder benigno em relação à amostra do pólo, enquanto estes últimos apresentaram significativamente maiores níveis de lócus de controle outros poderosos, crenças inibidoras da realização, afiliação no trabalho e afiliação geral.

As comparações entre as médias dos escores em cada uma das vinte variáveis motivacionais envolvidas nos modelos estudados (ver Melo, 2002) indicam que os sujeitos que se aproximam mais do modelo de cultura menor do que do modelo de cultura maior apresentam-se predominantemente como possuindo pelo menos uma das seguintes características: sexo masculino; mais velhos; casados; renda familiar mensal acima de três mil reais; renda individual mensal acima de quinhentos reais; seus pais e mães possuem maior nível de escolaridade; cursaram ensino médio em escola privada; declararam falar outras línguas; trabalham; são profissionais liberais, autônomos, proprietários ou sócios de empresas; ocupam cargo de supervisor, gerente ou superior; vivem em cidades maiores. Ao se considerarem os planos para o futuro, hábitos e preferências, os sujeitos responderam que pretendem continuar estudando, cursar mestrado ou doutorado, sair do país para estudar, montar um negócio próprio no futuro; gostam de ler, principalmente livros técnicos, jornais, revistas; compram mais livros; têm hábitos de ir ao cinema; navegam na internet; praticam esportes; fazem cursos paralelos; o programa preferido na televisão é o documentário; durante as férias, vão ao cinema, lêem, fazem cursos paralelos e navegam na internet.

\section{Estudos envolvendo professores e profissionais egressos}

Ao recolher dados junto a 119 professoras, com nível de escolaridade médio e superior, de escolas públicas municipais de Uberlândia, em Minas Gerais, Matos (1991) encontrou a presença de motivação de afiliação, poder e realização, nesta ordem, achados que se aproximam mais do modelo de cultura maior.

Dela Coleta e Dela Coleta (1997) estudaram 343 trabalhadores do sexo masculino, de organizações com diferentes portes e distintos ramos de atividade, de três estados da região sudeste, dos quais 115 ocupavam postos de chefia mais elevados e 228 eram chefes imediatos. Verificaram a prevalência de motivação de realização, seguida pela de afiliação e, por último, de poder, com maior variabilidade no grupo de chefes superiores e maior semelhança entre os três motivos no grupo de chefes imediatos.

Nesse estudo, os chefes superiores, em geral com nível superior de escolaridade em relação aos chefes imediatos, estes muitas vezes com pequena qualificação escolar, obtiveram significativo melhor desempenho em todas as variáveis (à exceção de poder benigno) constantes do modelo de cultura menor, apresentando-se com maior motivação de realização (tanto fixação de metas quanto instrumentação), internalidade, esperança ativa, otimismo, e significativa menor presença de crenças inibidoras da realização, crenças em outros poderosos, no acaso, poder explorador e afiliação, tanto no trabalho como na vida em geral.

Do estudo de Dela Coleta e Feltran (1998) participaram 406 professoras do ensino fundamental, de escolas do interior de Minas Gerais. Os resultados demonstraram possuírem os sujeitos níveis mais elevados de motivo de realização e de afiliação, auto-estima, internalidade, otimismo, níveis intermediários de esperança, e níveis mais baixos de motivação de poder, de crenças inibidoras da realização, em outros poderosos, no acaso. Tais dados indicam maior aproximação com o modelo de cultura menor, de superação do subdesenvolvimento, excetuando-se a sempre alta afiliação, que parece se caracterizar como um traço generalizado do povo brasileiro, inclusive daqueles indivíduos com maior 
escolaridade e mais voltados para o crescimento pessoal e a superação da situação em que se encontram.

Ao considerarem o tamanho das cidades em que vivem os sujeitos, os autores observaram diferenças significativas em cinco das seis variáveis tidas como exercendo influência negativa nos modelos motivacionais (crenças inibidoras da realização, crenças no acaso, em outros poderosos, poder explorador e afiliação geral), sendo o desempenho dos sujeitos das maiores cidades mais próximo do modelo de cultura menor do que daqueles de cidades de médio ou pequeno porte.

Dela Coleta e Feltran (1998) verificaram, ainda, que professoras com nível superior de escolaridade aproximavam-se mais do modelo de cultura menor do que aquelas com nível médio de escolaridade, mas não apresentavam diferenças quando estas últimas eram casadas e seus esposos eram portadores de nível superior de escolaridade, indicativos do importante papel desempenhado pelo ambiente motivacional do lar no desenvolvimento do indivíduo.

Dela Coleta e Mellão (2002) recolheram dados com 143 professores universitários de IES pública e privada, referentes a variáveis contidas nos modelos de cultura maior cultura menor propostos por Romero-Garcia (1991) e a medidas de comprometimento organizacional e satisfação no trabalho. Os dados mostraram que os professores apresentaram altos índices de fortaleza, excelência, otimismo, assertividade, internalidade, autoestima, esperança ativa e afiliação no trabalho e baixos índices de crenças inibidoras da realização, crenças em outros poderosos, crenças no acaso e motivação de poder explorador, dados que, à exceção do relativo alto nível médio em afiliação no trabalho, constante em todos os estudos conduzidos com amostras brasileiras, oferecem indicação de maior aderência do perfil motivacional desse grupo de professores ao modelo de cultura menor, associado ao crescimento pessoal, profissional e à superação do subdesenvolvimento.

Quando foram consideradas as variáveis positivas do modelo de cultura menor (excelência, auto-estima, assertividade, internalidade, esperança ativa, otimismo e fortaleza) e as variáveis de satisfação no trabalho, constatou-se uma predominância de coeficientes de correlação positivos entre elas, demonstrando que maior aderência ao modelo de cultura menor, por parte dos professores universitários, representa maior tendência de satisfação no trabalho, ocorrendo exatamente o contrário quando foram consideradas as variáveis negativas, indesejáveis, predominantes no modelo de cultura maior. Quanto ao comprometimento organizacional, quase não aparecem correlações significativas com as variáveis motivacionais, exceção aos escores do enfoque instrumental, que apresentaram correlações negativas com os escores nas variáveis desejáveis do modelo de cultura menor.

Psico-USF, v. 10, n. 1, p. 69-75, jan./jun. 2005

\section{Conclusões}

A revisão dos achados destas quase duas dezenas de publicações brasileiras envolvendo variáveis constantes dos modelos de cultura maior e de cultura menor, propostos por Romero-Garcia (1991), evidenciaram, em primeiro lugar, a consistência teórica dos modelos, sustentada pelas correlações positivas e significativas entre as variáveis desejáveis do modelo de cultura menor, o mesmo ocorrendo entre as variáveis indesejáveis e, por outro lado, correlações negativas e significativas entre as variáveis desejáveis, e as indesejáveis deste modelo (Melo, 2002; Pasti, 1997).

Os resultados médios dos sujeitos nos diversos estudos apresentam uma tendência a maior proximidade com o modelo de cultura menor, com altos escores em motivo de realização, internalidade, otimismo, esperança, assertividade, eficiência, excelência, fortaleza, autoestima, e baixos em motivação de poder, externalidade defensiva, externalidade autêntica, crenças inibidoras da realização, crenças negativas sobre o trabalho, restando como elemento destoante desta tendência os altos escores em afiliação geral e no trabalho, o que pode comprometer o comportamento das outras variáveis positivas constantes do modelo de cultura menor.

Os dados indicam também haver poucas dúvidas de que os sujeitos com educação superior tendem a apresentar resultados superiores nas variáveis desejáveis e inferiores nas variáveis indesejáveis do modelo de cultura menor, em relação àqueles com educação média, sugerindo que a educação superior seria um dos fatores capazes de levar as pessoas a adotar um conjunto de crenças positivas e favoráveis ao seu desenvolvimento pessoal.

Este fato, provavelmente, pode ser explicado por algumas das finalidades da formação universitária que apresentam relação direta com as variáveis aqui examinadas: busca de aprimoramento contínuo; não-aceitação de resultados que não sejam os melhores naquelas circunstâncias; tendência ao desenvolvimento de métodos e processos mais eficazes; crença de que sempre é possível fazer melhor, mais rápido e com mais economia; formulação de metas mais difíceis, exigentes e de longo prazo; responsabilidade pessoal pelos resultados; desenvolvimento de forças internas do indivíduo; preparação do cidadão para enfrentar adversidades da vida, para enfrentar as incertezas; espera por resultados positivos da ação individual; formação profissional ampla, profunda, de qualidade, atualizada; vinculação individual a contribuições para o aprimoramento da sociedade; formação ética e moral do cidadão.

É importante salientar que a presença de outros sujeitos com nível superior de escolaridade no lar (pai, mãe, esposo, esposa) parece desempenhar papel relevante nas condições desse ambiente motivacional, favorecendo a 
proximidade dos indivíduos criados neste contexto com o modelo de cultura menor, o mesmo ocorrendo quando o sujeito vive em cidades maiores e mais desenvolvidas ou possui renda familiar mais elevada.

Mas o dado mais interessante que emerge deste conjunto de estudos refere-se à relação sistemática entre a maior proximidade do modelo de cultura menor e melhor rendimento acadêmico, seja no ensino médio, superior ou nos processos seletivos para ingresso nas universidades (exames vestibulares), maiores níveis de satisfação no trabalho entre os professores universitários, bem como em diferenças marcantes nas condutas da vida diária e da vida acadêmica.

Por último, esta revisão mostra que a persistência na realização de estudos diversos e complementares envolvendo postulados de um bom modelo teórico em muito auxilia a compreensão do papel das variáveis psicossociais na determinação da conduta humana e, neste caso em particular, do papel da escolaridade no desenvolvimento do indivíduo como ser integral.

\section{Referências}

Almeida e Borges, D. M. (2001). Características psicossociais de universitários "exitosos" e "não exitosos": implicaçôes para a atividade docente. (Dissertação de Mestrado). Uberlândia: Centro Universitário do Triângulo.

Coopersmith, S. (1967). The antecedents of self-esteem. São Francisco: Freeman.

Dela Coleta, J. A. (1979). A escala de lócus de controle interno-externo de Rotter: um estudo exploratório. Arquivos Brasileiros de Psicologia, 31(4), 167-181.

Dela Coleta, J. A. (1988). Internalidad, auto-estima, valor incentivo de los estudios, necesidad de logro y rendimiento académico en tareas de máxima ejecución. Memorias Evemo, 2, 35-44.

Dela Coleta, J. A. (1989). Rendimento acadêmico em tarefas de realização máxima e variáveis psicossociais. Psicologia: Teoria e Pesquisa, 5(2), 177-189.

Dela Coleta, J. A. \& Dela Coleta, M. F. (1997). Algumas características motivacionais de trabalhadores brasileiros: a caminho da superação do subdesenvolvimento. Em A. Tamayo, J. E. Borges Andrade \& W. Codo (Org.). (1996). Trabalho, organizaçoes e cultura. (pp. 219232). São Paulo: Cooperativa de Autores Associados.

Dela Coleta, J. A. \& Feltran, R. C. S. (1998). Maestras en el interior de Brasil: crecimiento personal y papel de la escolaridad. Memorias Evemo, 7, 177-180.

Dela Coleta, J. A. \& Mellão, M. E. B. (2002). A motivação, o comprometimento e a satisfação no trabalho em grupos de professores universitários. Ícone-Educação, 8(1 e 2). 93-106.

Dela Coleta, J. A. \& Gomide, N. G. (2003). Características motivacionais de um grupo de estudantes de Administração. Anais da VII Reunião Anual de Ciências. Centro Universitário do Triângulo, Uberlândia, 79-80.

Dela Coleta, J. A, Almeida e Borges, D. M. \& Dela Coleta, M. F. (2003). Motivações sociais e rendimento acadêmico universitário. Revista Educação e Filosofia, 17(33), 47-62.

Dela Coleta, M. F. (1987). Escala Multidimensional de Lócus de Controle de Levenson. Arquivos Brasileiros de Psicologia, 39(2), 79-97.

Dela Coleta, M. F. (1988). Escala Levenson de locus de control con sujetos brasileños. Memorias Evemo, 2, Sección Motivaciones Sociales en América Latina, Mérida, Venezuela, 22-34.

Dela Coleta, M. F. \& Paula, C. M. (1998). Variáveis preditoras do rendimento acadêmico em estudantes universitários e secundaristas. Relatório de pesquisa CNPq/UFU.

Dela Coleta, M. F., Marcondes, A., Deolinda, D., Moreira, E. A., Alvarenga, L. S., Amaral, M. A. \& Santos, T. (2003). Variáveis psicossociais relacionadas com a busca de ingresso em curso superior. Sociedade Brasileira de Psicologia. Resumos de Comunicações Científicas, Belo Horizonte, 378.

Levenson, H. (1974). Activism and powerful others: Distinctions within the concept of internal-external locus of control. Journal of Personality Assessment, 38, 377-383.

Matos, L. A. L. (1991). Motivação para o trabalbo e stress em um grupo de professoras. (Monografia de Especialização). Uberlândia: Universidade Federal de Uberlândia.

McClelland, D. C. (1972). A sociedade competitiva: realização e progresso social. Rio de Janeiro: Expressão e Cultura.

Melo, M. A. S. (2002). Variáveis da cultura maior e da cultura menor em duas amostras de universitários de localidades com diferentes niveis de desenvolvimento. (Dissertação de Mestrado). Uberlândia: Centro Universitário do Triângulo.

Melo, M. A. S., Dela Coleta, J. A. \& Dela Coleta, M. F. (2003). Características motivacionais de amostras de universitários do interior do Brasil. Ícone - Educação, 9 (1 e 2), 183-197.

Pasti, M. J. (1997). Perfil motivacional do enfermeiro atuante em um hospital geral do interior paulista. (Dissertação de Mestrado). Ribeirão Preto: Escola de Enfermagem de Ribeirão Preto, Universidade de São Paulo. 
Romero-García, O. (1986). Investigación motivacional en el Laboratorio de Psicología de la Universidad de Los Andes. Universidad de Los Andes, Mérida, Venezuela. (publicación n. 80).

Romero-García, O. (1991). Crecimiento psicológico y motivaciones sociales. Mérida, Venezuela: ROGYA.

Rosemberg, M. (1965). Society and adolescent selfimage. Princeton, NJ: Princeton University Press.

Rotter, J. (1966). Generalized expectancies for internal versus external control of reinforcement. Psychological Monographs: General and Applied. Washington, 80.
Seligman, M. E. P. (1977). Desamparo: sobre depressão, desenvolvimento e morte. São Paulo: Hucitec, Ed. da Universidade de São Paulo.

Seligman, M. E. P. (1991). Learned optimism. New York: Alfred A. Knopf.

Souza, A. B. (2002). Motivação para o trabalho em amostras do interior do Brasil. (Dissertação de Mestrado). Uberlândia: Centro Universitário do Triângulo.

Recebido em janeiro de 2005 Reformulado em maio de 2005 Aprovado em junbo de 2005

Sobre os autores:

José Augusto Dela Coleta é psicólogo, mestre em Ergonomia, doutor em Psicologia Social e professor do Curso de Mestrado em Educação Superior do Centro Universitário do Triângulo, em Uberlândia, MG.

Marilia Ferreira Dela Coleta é psicóloga, mestre e doutora em Psicologia Social e professora do Instituto de Psicologia da Universidade Federal de Uberlândia, MG. 